\title{
Analogue simulation and analysis of non-circular gear reentry error
}

\author{
Jianqing Dai, Shengze Wang \\ School of mechanical engineering, Donghua University, Shanghai 201620, People's Republic of China
}

\begin{abstract}
The studies on non-circular gear cutting and using errors were generally based on theory of cylindrical gear errors, and the similarities and differences between cylindrical gear and non-circular gear were combined to study the change rule of non-circular gear contacting line and obtained corresponding conclusions. In this paper, from a different perspective, by starting from the non-circular gear engagement principle, on the basis of analyzing the concrete geometric error conditions, new type tooth profile algorithm routine is reasonably modified, and needed noncircular gear tooth profile error contrast figure is obtained. Furthermore, theoretical derivation is carried out by incorporating analytical method and contacting line incremental method, validity of computer simulation is verified, and the characterization of the tangential error characteristic code is obtained, Summed up the error sources for error genetic which possess enormous significance to direct practice generation.
\end{abstract}

\section{Introduction}

Non-circular gear in terms of its essence can be considered as a new type of institution which integrates the advantages of both cam and gear $[1,2]$. Non-circular gear mechanism not only inherited the numerous congenital advantage of the gear mechanism, but also has predictable characteristics of cam mechanism transmission rule. Therefore the non-circular gear is a kind of gear mechanism can achieve predictable variable speed ratio transmission [3].

Non-circular gear machining exist unique two errors, respectively are tool clamping error $[4,5]$ and reentry error of serrated knife, tool clamping error is closely related to the operator in practice, tool clamping error more belongs to the mistake error [6], and reentry error is related to tooth knife motion mechanism.

Both non-circular gear machining and cylindrical gear are subject to the basic principle of gear engagement, namely the serrated knife and gear do pure rolling, that is to say, translational distance of serrated knife should be in accordance with the curve that it passes through the pitch line. In the actual production, if the translational distance of serrated knife doesn't keep in accordance with the curve that it passes through the pitch line because of movement mechanism, therefore, tangential error generates at this moment. Due to the vast majority of tangential errors come from the eccentric movement of worm gear, essence is a harmonic gear error created by movement inharmonious between workpiece and tool, we only consider sine function of the turbine in a variety of harmonic errors.

$$
\Delta y=k \sin \theta
$$

Anticlockwise corner is positive value, reflectedin serrated knife translation motion is shown as follow.

$$
L^{\prime}=L+K \sin \theta
$$

$L$ is theoretical translation distance, $L^{\prime}$ is practical translation distance, and translational error conforms to sine function. According to relative motion principle, we considered that tooth germ doesn't move, serrated knife does reentry error movement.

\section{Reentry error model}

As shown in Figure 1, zero position of serrate knife just aligned the collinear with the zero position of work piece at a time of zero, in later actual production, according to relative motion principle, serrate knife generated $\Delta y$ error which changed according to the sine function along axis $y$, resulting in the unequal translation section of serrated knife like the curve that passed through the section curve, this is reentry error model.

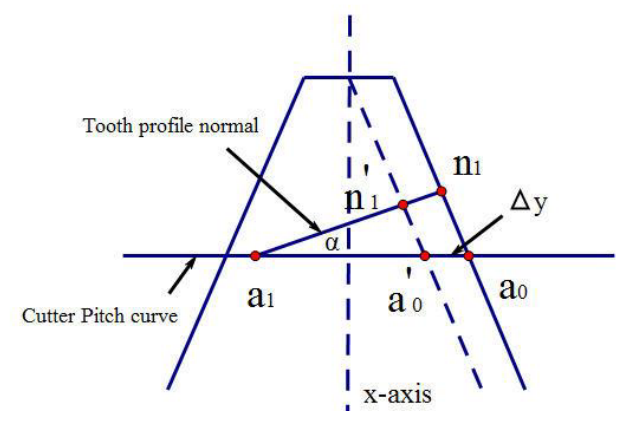

Figure 1. Reentry error model 


\section{Computer simulations}

We used beforehand programmed non-circular gear tooth profile algorithm program. We carried out a generative simulation of the serrated knife according to the operation condition of the zero alignment, and we got the theory tooth profile diagram without the error, then we added a loop statement to $\mathrm{x}$ coordinate value of serrated knife small coordinate system in subroutines for simulating $y$ coordinate figure remained immobility, to reflect reentry error conditions, then running the program again to get the tooth profile pattern when reentry error appeared. Finally, two pictures were combined in the same coordinate system to form Figure 2- Figure 5, that is, reentry error tooth profile comparison diagram (The circles are theory tooth profile, the stars are the tooth profile error).

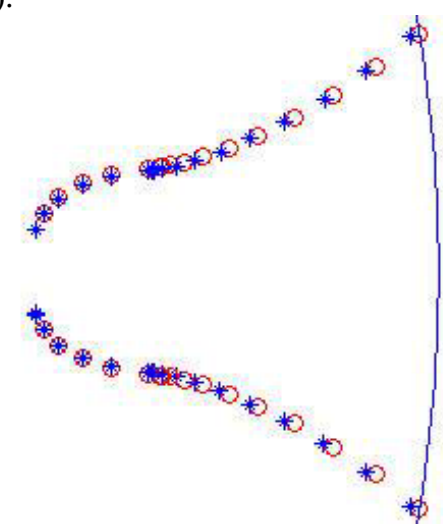

Figure 2. Comparation of initial alveoli reentry error tooth profile

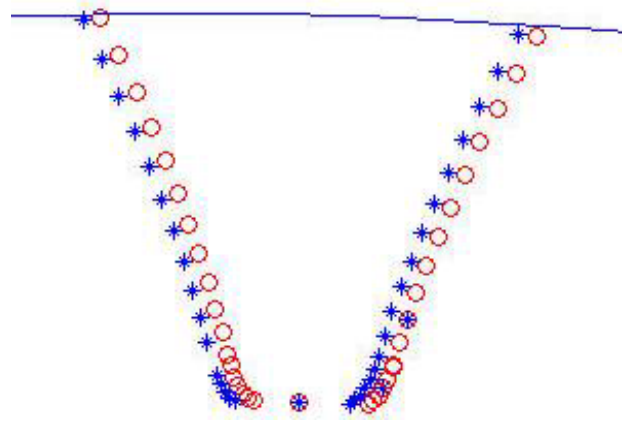

Figure 3. Comparation of reentry error tooth profile in short axis direction

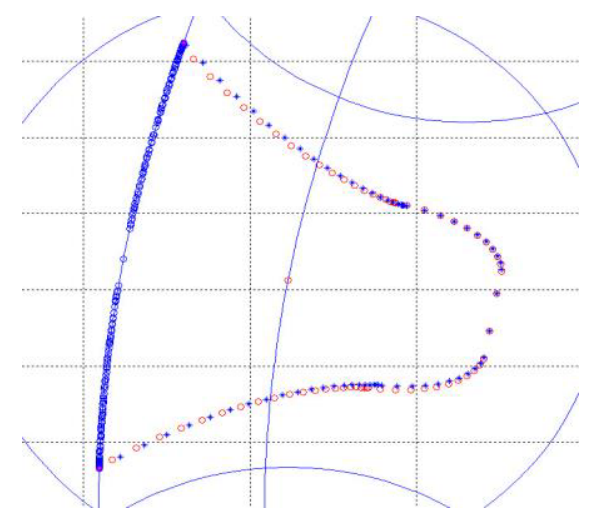

Figure 4. Comparation of reentry error tooth profilein long axis negative direction
Figure 2 and Figure 4 show the initial positions, that is, comparations of alveolar reentry error tooth profiles in long axis positive direction and short axis direction. Figure 3 and Figure 5 show the comparations of alveolar reentry error tooth profiles nearby the non-circular gear short axis positive direction and negative direction.

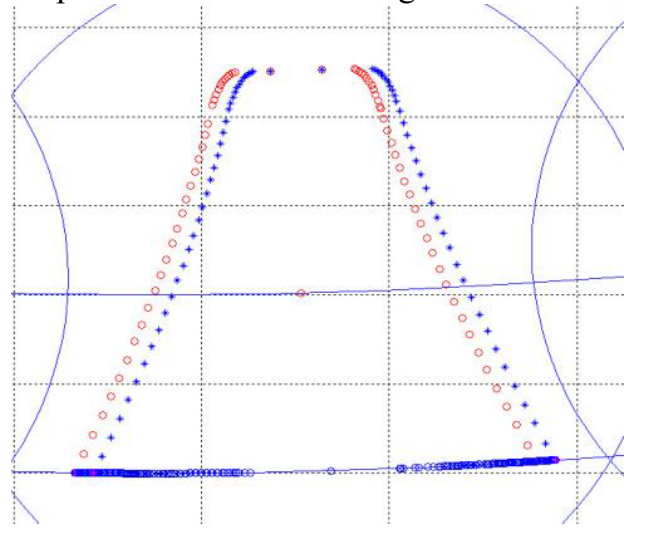

Figure 5. Comparation of reentry error tooth profile in short axis negative direction

Small red circles shows the theory tooth profile without error in from Figure 2 to Figure 5, while blue stars are tooth profile graphs that have reentry error.

\section{Simulation conclusions}

We can observe from the picture that the tooth profile deviation of tooth profile comparation Figure 2 and Figure 4 are not obvious, and the tooth profile excursion of the tooth profile contrast diagrams Figure 3 and Figure 5 are the most obvious. Tooth profile deviation of tooth profile contrast Figure 3 is toward to counter-clockwise direction, the tooth profile deviation of tooth profile contrast Figure 5 is toward to the clockwise direction, roughly the same size, in the opposite direction.

The formed tooth profile and the theory tooth profile after the excursion of tooth profile contrast diagrams Figure 3 and Figure 5 are different, that is, error tooth profile isn't the simple equidistant line of theory tooth profile.

The pitch curve of serrated knife tangent in the normal direction and alveoli bottom are basic coincidence, there is no obvious change, and that is, the radial error is zero.

\section{Theoretical verification}

In order to set the tooth profile normal distribution regulation of the non-circular beforehand for convenience, it's determined by the following [7].

$$
\lambda-\mu=\text { constant }
$$

$\lambda$ is the angle of section curve diameter and corresponding tooth profile normal, $\mu$ is the angle of section curve diameter and the tangent line positive direction.

Because of the special nature of noncircular gear pitch curve, leading to correspond exactly to the correlation 
does not exist between the theoretical tooth profile and tooth profile error, according to the principle of gear meshing in Figure 1 has two non circular gear to find the way. The first kind is to generate the error after $a_{1} n_{1}$ does not move, its new tooth shape intersect at $n_{1}^{\prime}$ point, points $n_{1}$ and point $n_{1}^{\prime}$ to form the first class correlation point; The second kind is to produce the error after $a_{1} n_{1}$ is also corresponding to move, the length of the same, and its new tooth shape intersect at $n_{1}^{\prime}$ point, points $n_{1}$ and point $n_{1}^{\prime}$ to form the first class correlation point, here we first study the first type of correlation point. Fom Figure 1 , we can get

$$
a_{1} n_{1}=a_{0} a_{1} * \cos \alpha
$$

According to the theory of engagement, when the instantaneous rotation center for serrated knife is $a_{1}$ point, $n_{1}$ point of rack tooth profile corresponds with $n$ point on conjugate tooth profile, when serrated knife offsets to the left for $\Delta x$, the point of $a_{1}$ respectively corresponds with $n_{1}$ point on rack tooth profile and $n_{1}^{\prime}$ point on conjugate tooth profile, on the other hand, the section curve from the instantaneous rotation center to original point is determined by the following conditions.

$$
S_{1}=a_{0} a_{1}
$$

The serrated knife excursions also comply with the above rule, but the arc length changes. In Figure 2, vector equation of the tooth profile can be expressed by the following $[8,9]$.

$$
\overrightarrow{r_{f}}=\overrightarrow{r_{g}}+\overrightarrow{a n}
$$

Right tooth profile equationis obtained when converting into rectangular coordinates.

$$
\left\{\begin{array}{l}
x_{R}=r_{g} \cos \theta+\text { an } \cos (\theta+u-\alpha) \\
y_{R}=r_{g} \sin \theta+a n \sin (\theta+u-\alpha)
\end{array}\right.
$$

Left tooth profile equation

$$
\left\{\begin{array}{l}
x_{L}=r_{g} \cos \theta+a^{\prime} n^{\prime} \cos (\theta+u+\alpha-\pi) \\
y_{L}=r_{g} \sin \theta+a^{\prime} n^{\prime} \sin (\theta+u+\alpha-\pi)
\end{array}\right.
$$

The tooth profile normal length is acquired by the following

$$
\begin{array}{r}
a n=a_{0} a \cos \alpha_{u}=S * \cos \alpha_{u} \\
a^{\prime} n^{\prime}=a_{0}^{\prime} a^{\prime} \cos \alpha_{u}=S * \cos \alpha_{u}
\end{array}
$$

The analytical formula for theory tooth profile is above one offset $\Delta y$ in Figure 1 is as following after theory tooth profile

$$
a_{0} a_{0}^{\prime}=\Delta y
$$

Simplified as error vector quantity

$$
a_{1} n_{1}=a_{0} a_{1} \cos \alpha_{u}
$$

Formula is got after being converted into rectangular coordinate

$$
\left\{\begin{array}{l}
x_{n}^{\prime}=x_{n} \mp a_{0} a_{0}^{\prime} \cos \alpha_{u} \operatorname{tg} \alpha_{u} \\
y_{n}^{\prime}=y_{n} \mp a_{0} a_{0}^{\prime} \cos \alpha_{u} \operatorname{ctg} \alpha_{u}
\end{array}\right.
$$

Due to the preceding error model, we can get the tool clamping error is an actual case of reentry error. Tool clamping error is got when $\Delta y$ is a constant value, when $\Delta y$ is a variate value, in principle, we need to determine the relevant $\Delta y$ value that each gear cutting angle of tooth profile should correspond with, and consider the variate value $\Delta y$ as the serrated knife offset of corresponding angle. In reentry error conditions, offset $\Delta y$ changes follow the change of the cutting angle and comply with the sine function law $\Delta y=k \sin \theta$, here, in order to simplify the problem, and highlight the contrast effect, we set the variation cycle of $\Delta y$ as $T=2 \pi$, with the movement function that changes with angle, we can conveniently find the offset $\Delta y$ that every knives of reentry error correspond, again in the program, create a loop statement of serrated knife offset that changes with angle and $\Delta y$, it can simulate the tooth profile diagram of the reentry errorand summarize the error code of reentry error from it. Actually, if the offset $\Delta y$ changes according to other rules, we only need to focus on the regular function, although the final result is different, but the nature of the problem is still the same.

\section{Theoretical explanations}

We can learn from Figure 1 that tangential error changes generally in accordance with $\Delta y$ and it is generally in accordance with the law of sine function, it also explains why tangential error is small when it is the long axis, and short axis is larger and the offset changes with sine function. In the range of $0 \sim \pi$, tangential error make the tooth profile migrate anticlockwise, in the range of $\pi \sim 2 \pi$, tangential error make the tooth profile migrate clockwise, and because the values of $\Delta y$ Maximum value are the same, tooth profile migrations are about the same size, and in the opposite direction.

By Eq. (13), we know due to the various curvature radiuses of points on the section curve, leading to the each included angles $u$ of each point radius vector and the tangent line are not identical, the vectorial direction of the offset is not exactly the same, so it can be proved the correctness of the simulation conclusion.

From Figure 1 that cutter pitch curve and ellipse pitch curve tangent, and no method to offset, so the simulation results will be no radial error.

\section{Experiment and classification}


From the above analysis, we know the reason and theoretical explanation of the tool setting error, In the actual test, we can process the non-circular gear solid model which is shown in Figure 6 through the rapid prototyping system, Using the RoyalArm RA315 measuring arm (measurement accuracy of $0.05 \mathrm{~mm}$ ) which shows in Figure 7 on the key points of non-circular contrate profile actual coordinates value and theoretical coordinate value difference judgment whether to produce tool setting error or other tangential error.

The experimental method is as follows

(1) Non-circular gear processing must have a zero, that is, the initial processing position. In the model above the left end elliptic focus as the origin of coordinates, the right level for the positive direction of axis, vertical axis direction, establish coordinate system.

(2) Because of the large number of non-circular gear tooth, large quantities of one of the measurement of each tooth space for all positions of the geometric point in the actual production is not acceptable, so we at the end of the ellipse long axis and short axis at both ends of each selected a tooth, coordinate measurement of tooth top apex and tooth root value.

(3) The actual coordinates the collection value and coordinate theory are compared to the values, the zero cogging data must be collected, the case of zero cogging in long axis is the direction and the results are shown in Table 1 .

(4) According to the results of the comparison of the results in Figure 9 can be classified to learn about the causes and sources of error. Because this article is only relates to cut to the field of error, Figure 9 relates to the radial error and the geometric deviation theory proof in my other are published articles are presented in detail. This only reference related to cut to the part of the conclusion of the error.

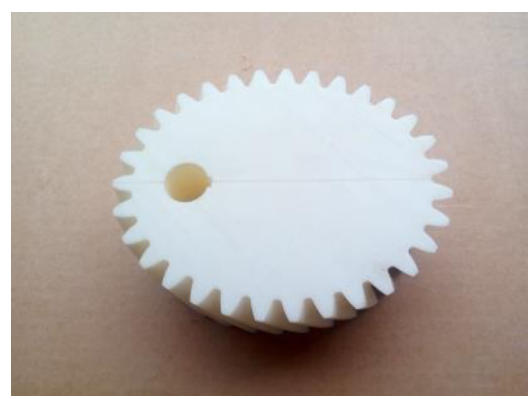

Figure 6. Solid model of non-circular gear

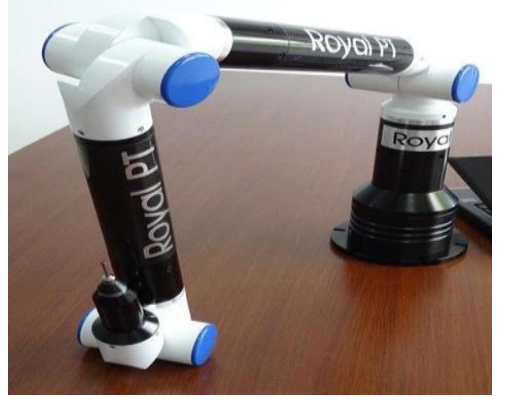

Figure 7. Joint arm measuring instrument

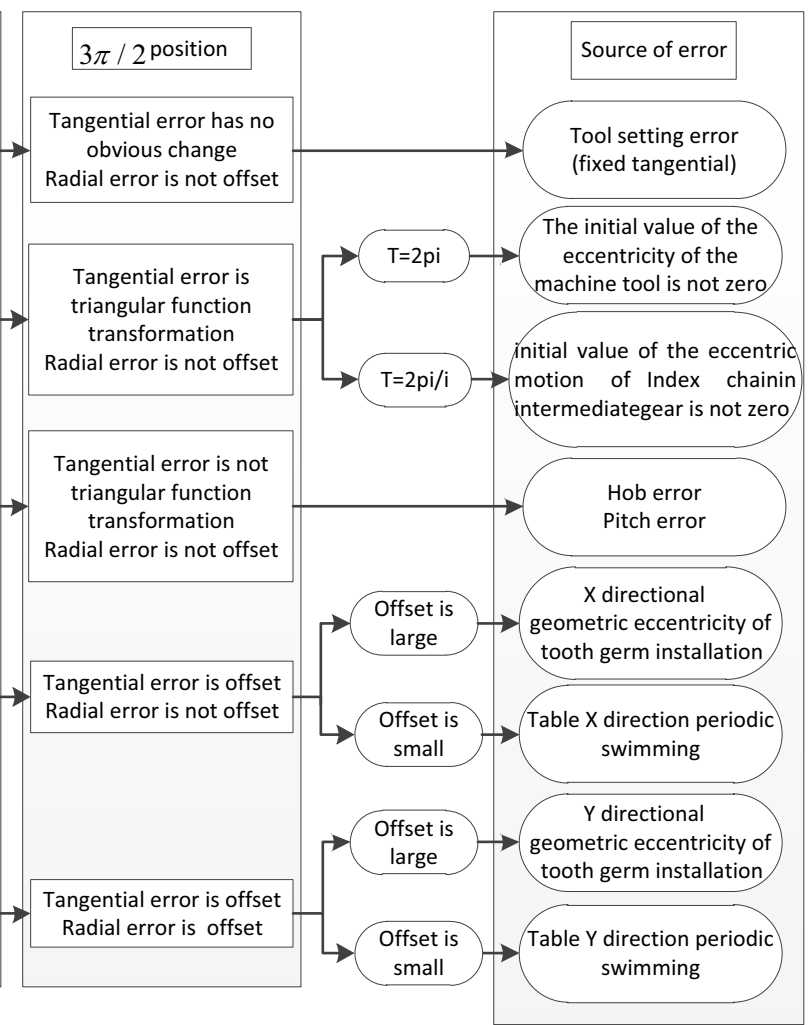

Figure 8. Tangential error factors of error genetic diagram 


\section{ICMMR 2016}

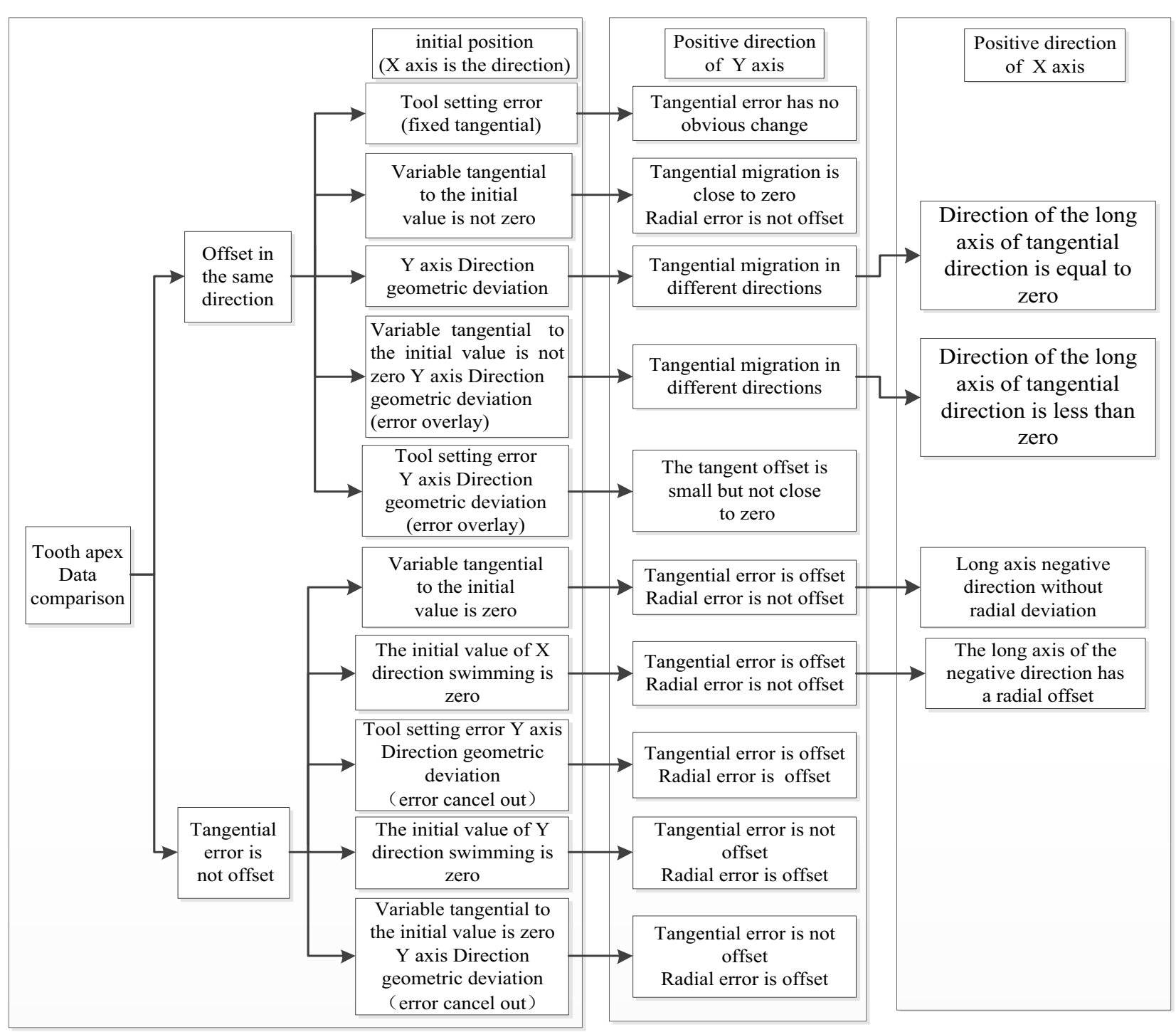

Figure 9. Characterization of characteristic code

Table 1. Tooth profile data sheet

\begin{tabular}{|c|c|c|c|c|c|c|c|c|}
\hline & \multicolumn{2}{|c|}{$\begin{array}{l}\text { Long axis normal } \\
\text { direction }\end{array}$} & \multicolumn{2}{|c|}{$\begin{array}{c}\text { Short axis normal } \\
\text { direction }\end{array}$} & \multicolumn{2}{|c|}{$\begin{array}{l}\text { Long axis negative } \\
\text { direction }\end{array}$} & \multicolumn{2}{|c|}{$\begin{array}{l}\text { Short axis negative } \\
\text { direction }\end{array}$} \\
\hline $\begin{array}{l}\text { X- theoretical tooth } \\
\text { top apex }\end{array}$ & 35.2954 & 35.2954 & 16.7847 & 14.3307 & -5.4062 & -5.9078 & 14.3250 & 16.7847 \\
\hline $\begin{array}{l}\text { Y- theoretical tooth } \\
\text { top apex }\end{array}$ & -1.4003 & 1.4003 & 13.9980 & 13.9331 & 3.0883 & 0.3393 & -13.9980 & -13.9330 \\
\hline $\begin{array}{l}\text { X- actual tooth top } \\
\text { apex }\end{array}$ & 35.30 & 35.30 & 16.70 & 14.45 & -5.40 & -5.90 & 14.40 & 16.90 \\
\hline $\begin{array}{l}\text { Y- actual tooth top } \\
\text { apex }\end{array}$ & 1.30 & 1.45 & 13.95 & 14.00 & 3.00 & 0.25 & -14.00 & -13.95 \\
\hline $\begin{array}{l}\mathrm{X} \text { - theoretical } \\
\text { tooth of root }\end{array}$ & \multicolumn{2}{|c|}{33.0952} & \multicolumn{2}{|c|}{15.4782} & \multicolumn{2}{|c|}{-3.5371} & \multicolumn{2}{|c|}{15.4745} \\
\hline $\begin{array}{l}\text { Y- theoretical } \\
\text { tooth of root }\end{array}$ & \multicolumn{2}{|c|}{0} & \multicolumn{2}{|c|}{11.7433} & \multicolumn{2}{|c|}{1.3606} & \multicolumn{2}{|c|}{-11.7436} \\
\hline $\begin{array}{l}\text { X-actual } \\
\text { tooth of root }\end{array}$ & \multicolumn{2}{|c|}{33.10} & \multicolumn{2}{|c|}{15.40} & \multicolumn{2}{|c|}{-3.55} & \multicolumn{2}{|c|}{15.55} \\
\hline $\begin{array}{l}\text { Y-actual } \\
\text { tooth of root }\end{array}$ & \multicolumn{2}{|c|}{0.10} & \multicolumn{2}{|c|}{11.75} & \multicolumn{2}{|c|}{1.25} & \multicolumn{2}{|c|}{-11.70} \\
\hline
\end{tabular}


In Ttable 1 at the coordinate data of zero tooth apex the conclusion can be obtained that the tooth profile is not offset, and the tangential direction is shifted in the same direction. From Figure 9, we know that this phenomenon may have five reasons. Tool setting error, the initial cutting error is not zero; the direction of geometrical deviations, the initial cutting error is zero and the direction of the geometric deviation of these two factors superimposed; tool setting error and the orientation geometric deviation of these two factors superimposed. The data of the short axis normal direction can be obtained in the radial direction, and there is no significant change in the tangential migration, from Figure 9 we know the model belongs to the typical causes of tool setting error.

From this example we get the test method of the origin of the tangential error and the characterization of the genetic code. In the actual production we are more concerned about how to quickly troubleshoot what are the process error factors that cause the error, so in Figure 9 based combined with common cause of cut to error of process factors are summarized Figure 8 non-circular gear tangential error factors of the error genetic diagram. So in Figure 9 based on combination of common cause of cutting error of process factors to get Figure 8 noncircular gear tangential error factors of the error genetic diagram. This will provide a powerful tool for the production of the cause of the problem quickly.

\section{Conclusions}

Via the system analysis and the theoretical proof of the computer simulation results for non-circular gear reentry error, we may obtain the conclusion that the reentry error is a tangential error that changes by trigonometric function, it's beneficial for the research complement of the non-circular gear error system and lay a solid foundation for the subsequent individual error of other conditions, and got the condition code of the reentry error excursion, providing theory support for timely finding out the cause of the gear errors when the factory inspection, thus improve the machining precision of the non-circular gear.

\section{References}

1. Baoni, L. I., Zhang, Y. The application and development trend of noncircular gear. Machine Tool \& Hydraulics. 4, 098 (2008)

2. Guoxing Sun, Chuanqiong Sun, Yongde Liu. The application prospect of non-circular gear on infinitely variable speed, Equipment maintenance technology, 1, 1-4 (2009)

3. Xueqiang Gao. Transmission technology overview of non-circular gear, Mechanical drive, 3, 5-8 (2003)

4. Huiping Yan, Chibing Hu, Limei Liu. The research of straight tooth non-circular gear hobbing machining error analysis, Mechanical drive, 4,18-20 (2007)

5. Fangyong Tian, Chibing $\mathrm{Hu}$, Yancang Jiang. An automatic tool setting method of non-circular gear hobbing, Mechanical research and application, 4, 4143 (2010)

6. Liqun $\mathrm{Wu}$, Hongxia $\mathrm{Bi}$. The determination of axial special-shaped gear NC hobbing machining adjusted cutter point coordinates, Coal mine machinery, 6, 109-112 (2008)

7. Yongping Liu, Peng Wang, Jia Li. The elliptical gear tooth profile design via analytical method, Machinery Design and Manufacture, 2, 214-216 (2015)

8. Fusheng Li. Non-circular gear, China Machine Press (1975)

9. Mingtong Wang. Involute gear accuracy, China Machine Press (1983) 